\title{
DISCONTINUITY OF A DEGENERATING ESCAPE RATE
}

\author{
LAURA DEMARCO AND YÛSUKE OKUYAMA
}

\begin{abstract}
We look at degenerating meromorphic families of rational maps on $\mathbb{P}^{1}$ - holomorphically parameterized by a punctured disk - and we provide examples where the bifurcation current fails to have a bounded potential in a neighborhood of the puncture. This is in contrast to the recent result of FavreGauthier that we always have continuity across the puncture for families of polynomials; and it provides a counterexample to a conjecture posed by Favre in 2016. We explain why our construction fails for polynomial families and for families of rational maps defined over finite extensions of the rationals $\mathbb{Q}$.
\end{abstract}

\section{INTRODUCTION}

Let $f_{t}$ be a holomorphic family of rational maps on $\mathbb{P}^{1}$ of degree $d>1$, parameterized by the punctured unit (open) disk $\mathbb{D}^{*}=\{t \in \mathbb{C}: 0<|t|<1\}$, and assume that the coefficients of $f_{t}$ extend to meromorphic functions on the unit disk $\mathbb{D}=\{t \in \mathbb{C}:|t|<1\}$. Let $a: \mathbb{D} \rightarrow \mathbb{P}^{1}$ be a holomorphic map. In this article, we examine the potential function $g_{f, a}$ on $\mathbb{D}^{*}$ (having the order $o(\log |t|)$ as $t \rightarrow 0$ ) for the bifurcation measure associated to the pair $(f, a)$. Our main result is that this potential function does not necessarily extend continuously across the puncture at $t=0$.

The question of continuous extendability of $g_{f, a}$ across $t=0$ arose naturally in the study of degenerating families of rational maps, and specifically in the context of equidistribution questions and height functions associated to the family $f_{t}$; see, e.g., $\mathrm{BD}, \mathrm{Fa} 2$. Continuity of the potential at $t=0$ was required to apply certain equidistribution theorems on arithmetic varieties (as in the proofs of the main results of [BD, DM, FG1, GY] and others). Moreover, when $a(t)$ parameterizes a critical point of $f_{t}$, the bifurcation measure and its potential are related to the structural stability of the family $f_{t}[\mathrm{De1}, \mathrm{DF}$. It is well known that continuity holds when $f_{t}$ has a uniform limit on the whole $\mathbb{P}^{1}$ as $t \rightarrow 0$, for any choice of $a$. It is also true when $f_{t}$ is any family of polynomials with coefficients meromorphic in $t$, again for any choice of $a$ [FG2].

To formulate the problem and our construction more precisely, we will work with $f_{t}$ in homogeneous coordinates: assume that we are given a family of homogeneous polynomial maps $\tilde{f}_{t}: \mathbb{C}^{2} \rightarrow \mathbb{C}^{2}$ of degree $d$, where the coefficients are holomorphic functions on the entire disk $\mathbb{D}$ such that for every $t \in \mathbb{D}^{*}, \tilde{f}_{t}^{-1}(0,0)=\{(0,0)\}$ and $\tilde{f}_{t}$ projects to $f_{t}$ on $\mathbb{P}^{1}$. There exists a continuous plurisubharmonic escape rate

$$
G_{\tilde{f}}: \mathbb{D}^{*} \times\left(\mathbb{C}^{2} \backslash\{(0,0)\}\right) \rightarrow \mathbb{R}
$$

Received by the editors October 4, 2017, and, in revised form, January 31, 2018.

2010 Mathematics Subject Classification. Primary 37F45; Secondary 37P30.

This research was partially supported by JSPS Grant-in-Aid for Scientific Research (C), 15K04924, and the National Science Foundation DMS-1600718. 
such that for each fixed $t \in \mathbb{D}^{*}$, the current $d d^{c} G_{\tilde{f}}(t, \cdot)$ on $\mathbb{C}^{2} \backslash\{(0,0)\}$ projects to the measure of maximal entropy of $f_{t}$ on $\mathbb{P}^{1}$ [HP, FS. Given a holomorphic lift of $a$ to $\tilde{a}: \mathbb{D} \rightarrow \mathbb{C}^{2} \backslash\{(0,0)\}$, we may write

$$
G_{\tilde{f}}(t, \tilde{a}(t))=\eta \log |t|+g_{f, a}(t)
$$

where $\eta \in \mathbb{R}$ represents a "local height" for the pair $(f, a)$, and the function $g_{f, a}$ on $\mathbb{D}^{*}$ satisfies

$$
g_{f, a}(t)=o(\log |t|)
$$

as $t \rightarrow 0$ De4; see $\$ 2.1$ The value of $\eta$ and the subharmonic function $g_{f, a}$ depend on the choices of $\tilde{f}$ and $\tilde{a}$, but $g_{f, a}$ is uniquely determined up to the addition of a harmonic function on $\mathbb{D}^{*}$ which is bounded near $t=0$. The Laplacian $\mu_{f, a}=$ $\frac{1}{2 \pi} \Delta g_{f, a}$ on $\mathbb{D}^{*}$ is the bifurcation measure associated to the pair $(f, a)[\mathrm{DF}, \S 3]$.

It turns out that the function $g_{f, a}$ is always bounded from above near $t=0$ (Lemma 2.1). In this article, we construct examples of pairs $(f, a)$ that satisfy

$$
\limsup _{t \rightarrow 0} g_{f, a}(t)=-\infty
$$

to show that it need not be bounded from below, so in particular does not extend continuously across $t=0$. In our examples, the maps $f_{t}$ will converge to a rational map $\varphi$ on $\mathbb{P}^{1}$ of degree $<d$ as $t \rightarrow 0$ locally uniformly on $\mathbb{P}^{1} \backslash H$, where $H$ is a nonempty finite set. The idea of the construction is to choose $\varphi$ and $a$ so that some sequence of iterates $\varphi^{n_{j}}(a(0))$ accumulates fast on $H$ as $n_{j} \rightarrow \infty$.

Furthermore, choosing $a(t)$ to parameterize a critical point of the family $f_{t}$, we obtain a counterexample to the continuity statement in [Fa2, Conjecture 1], in proving:

Theorem 1.1. For every integer $d>1$, there exists a holomorphic family $f_{t}$ of rational maps on $\mathbb{P}^{1}$ of degree $d$, parameterized by $t \in \mathbb{D}^{*}$, whose coefficients extend to meromorphic functions on $\mathbb{D}$ but for which the bifurcation current associated to the family $f_{t}$ fails to have a bounded potential in any punctured neighborhood of $t=0$.

Remark. It will be clear from the proof that the family $f_{t}$ can be chosen to be algebraic, in the sense that it extends to define a holomorphic family parameterized by $t$ in a quasiprojective curve $X$, with coefficients that are meromorphic on a compactification of $X$.

The bifurcation current associated to the family $f_{t}$ is equal to the Laplacian of the continuous and subharmonic function $t \mapsto L\left(f_{t}\right)$ on $\mathbb{D}^{*}$, where for each $t, L\left(f_{t}\right)$ is the Lyapunov exponent of $f_{t}$ with respect to its unique measure of maximal entropy. For more details on $L\left(f_{t}\right)$ and its relationship to $g_{f, a}$, see Section 3 .

The construction of examples of pairs $(f, a)$ for which $g_{f, a}$ fails to extend continuously across $t=0$ is laid out in Section [2. Our use of the Baire category theorem in the construction is similar to that of [Fa1, Example 4], $\mathrm{Bu}$, or [DG] in the context of higher-dimensional (bi)rational maps. In Section 3 , we give the proof of Theorem 1.1. In Section 4, we comment on why the strategy for producing these examples fails for families of polynomials and for rational maps on $\mathbb{P}^{1}$ defined over $\overline{\mathbb{Q}}$. We expect that a continuous extension of $g_{f, a}$ to $\mathbb{D}$ always exists when the pair $(f, a)$ is algebraic and defined over $\overline{\mathbb{Q}}$, as is known for algebraic families of elliptic curves [Si2, Theorem II.0.1] and therefore also for Lattès maps on $\mathbb{P}^{1}$ [DM, Proposition 3.4]; see also [JR, Theorem A] in the context of (bi)rational maps 
in dimension 2. The bifurcation current associated to a family $f$ was introduced in De1; its properties at infinity in the moduli space of quadratic rational maps (related to our Theorem 1.1) were studied in BG].

\section{A RECIPE FOR DISCONTINUITY}

In this section, we construct the examples for which $g_{f, a}$ fails to extend continuously to the disk $\mathbb{D}$.

2.1. The potential is bounded from above. Suppose we are given a family of homogeneous polynomial maps $\tilde{f}_{t}: \mathbb{C}^{2} \rightarrow \mathbb{C}^{2}$ of degree $d>1$, where the coefficients are holomorphic functions on the entire disk $\mathbb{D}$ and such that for every $t \in \mathbb{D}^{*}$, we have $\tilde{f}_{t}^{-1}(0,0)=\{(0,0)\}$ so $\tilde{f}_{t}$ projects to a rational map $f_{t}$ on $\mathbb{P}^{1}$ of degree $d$. We let $\tilde{a}: \mathbb{D} \rightarrow \mathbb{C}^{2} \backslash\{(0,0)\}$ be any holomorphic map, and let $a: \mathbb{D} \rightarrow \mathbb{P}^{1}$ be its projection. For each $n \in \mathbb{N}$, there is a unique nonnegative integer $o_{n}$ so that

$$
F_{n}(t):=t^{-o_{n}} \tilde{f}_{t}^{n}(\tilde{a}(t))
$$

is a holomorphic map from $\mathbb{D}$ to $\mathbb{C}^{2} \backslash\{(0,0)\}$. Choose any norm $\|\cdot\|$ on $\mathbb{C}^{2}$. The function $g_{f, a}$ on $\mathbb{D}^{*}$ defined by (1.1) is the locally uniform limit on $\mathbb{D}^{*}$ of the sequence of continuous and subharmonic functions

$$
g_{n}(t):=\frac{1}{d^{n}} \log \left\|F_{n}(t)\right\| \quad \text { on } \mathbb{D},
$$

as $n \rightarrow \infty$, and the value $\eta$ of (1.1) is given by

$$
\eta=\lim _{n \rightarrow \infty} \frac{o_{n}}{d^{n}}
$$

as explained in [De4, §3]. Note, in particular, that the function $g_{f, a}$ is continuous and subharmonic on $\mathbb{D}^{*}$.

The following observation is not required in this section, but it will be useful in Section 3 .

Lemma 2.1. The function $g_{f, a}$ is bounded from above on $\{0<|t| \leq r\}$ for every $r \in(0,1)$.

Proof. Fix any $r \in(0,1)$. We know that $\lim _{n \rightarrow \infty} g_{n}=g_{f, a}$ uniformly on the circle $C_{r}=\{|t|=r\}$. Let $M_{r}=\max _{C_{r}} g_{f, a}$. Then, for all $n$ large enough, we have $g_{n} \leq M_{r}+1$ on $C_{r}$. As the functions $g_{n}$ are subharmonic on $\mathbb{D}$, we also have $g_{n} \leq M_{r}+1$ on the disk $\{|t| \leq r\}$ for all $n$ large enough. It follows that $g_{f, a} \leq M_{r}+1$ on the punctured disk $\{0<|t| \leq r\}$.

Remark. As an immediate consequence of Lemma 2.1. the function $g_{f, a}$ extends to a subharmonic function on the disk $\mathbb{D}$, though its value at $t=0$ may be $-\infty$. In this article, however, we will keep the domain of the definition of $g_{f, a}$ as the punctured disk $\mathbb{D}^{*}$.

2.2. The ingredients for discontinuity. Let $\varphi \in \mathbb{C}(z)$ be a rational map on $\mathbb{P}^{1}$ of degree $e \geq 1$, and suppose that there is a point $a_{0} \in \mathbb{C}$ such that $\#\left\{\varphi^{n}\left(a_{0}\right): n \in\right.$ $\mathbb{N}\}=\infty$ and that $\omega_{\varphi}\left(a_{0}\right) \cap\left\{\varphi^{n}\left(a_{0}\right): n \in \mathbb{N}\right\} \neq \emptyset$, where

$$
\omega_{\varphi}\left(a_{0}\right):=\bigcap_{N \in \mathbb{N}} \overline{\left\{\varphi^{n}\left(a_{0}\right): n>N\right\}}
$$

is the $\omega$-limit set of $a_{0}$ under $\varphi$. Then there exists $N_{0} \in \mathbb{N}$ so that $\left\{\varphi^{n}\left(a_{0}\right): n \geq N\right\}$ is dense in $\omega_{\varphi}\left(a_{0}\right)$ for all $N \geq N_{0}$. 
Let $\left\{r_{n}\right\}$ be any sequence in $\mathbb{R}_{>0}$ decreasing to 0 as $n \rightarrow \infty$, which will be chosen appropriately later. It follows that the set

$U_{N}\left(a_{0},\left\{r_{n}\right\}\right):=\left(\bigcup_{n \geq N}\left\{z \in \omega_{\varphi}\left(a_{0}\right):\left[z, \varphi^{n}(a)\right]<r_{n}\right\}\right) \backslash\left\{a_{0}, \varphi\left(a_{0}\right), \ldots, \varphi^{N-1}\left(a_{0}\right)\right\}$

is open and dense in $\omega_{\varphi}\left(a_{0}\right)$ for all $N \geq N_{0}$. Here $[\cdot, \cdot]$ denotes the chordal distance on $\mathbb{P}^{1}$. Therefore, by the Baire category theorem,

$$
B_{\varphi}\left(a_{0},\left\{r_{n}\right\}\right):=\bigcap_{N \geq N_{0}} U_{N}\left(a_{0},\left\{r_{n}\right\}\right)
$$

is dense in $\omega_{\varphi}\left(a_{0}\right)$.

Fix any $h \in B_{\varphi}\left(a_{0},\left\{r_{n}\right\}\right) \cap \mathbb{C}$. Then $\varphi^{n}\left(a_{0}\right) \neq h$ for all $n \in \mathbb{N} \cup\{0\}$, and there is a sequence $n_{j} \rightarrow \infty$ such that

$$
0<\left[\varphi^{n_{j}}\left(a_{0}\right), h\right]<r_{n_{j}}
$$

for all $j \in \mathbb{N}$.

We consider the family

$$
f_{t}(z):=\varphi(z) \cdot \frac{z-h-\varepsilon t}{z-h+\varepsilon t}
$$

parameterized by $t \in \mathbb{D}^{*}$, where $\varepsilon>0$ is chosen so that $\varphi$ has neither zeros nor poles in the set $\{z: 0<|z-h|<\varepsilon\}$. Thus, $f_{t}$ defines a holomorphic family of rational maps of degree $d:=e+1>1$. As $t \rightarrow 0$, the maps $f_{t}$ converge locally uniformly to $\varphi$ on $\mathbb{P}^{1} \backslash\{h\}$.

\subsection{An unbounded escape rate. Now set}

$$
r_{n}=\exp \left(-n d^{n+1}\right)
$$

for each $n \in \mathbb{N}$. Working on $\mathbb{C}^{2}$, we define

$$
\tilde{f}_{t}(z, w):=(P(z, w)(z-(h+\varepsilon t) w), Q(z, w)(z-(h-\varepsilon t) w))
$$

for all $t \in \mathbb{D}$, where $P$ and $Q$ are homogeneous polynomials of degree $e=\operatorname{deg} \varphi$ such that $\varphi(z)=P(z, 1) / Q(z, 1)$. Let $\tilde{a}: \mathbb{D} \rightarrow \mathbb{C}^{2} \backslash\{(0,0)\}$ be any holomorphic map such that $\tilde{a}(0)=\left(a_{0}, 1\right)$, and let $a: \mathbb{D} \rightarrow \mathbb{P}^{1}$ be its projection to $\mathbb{P}^{1}$.

Choose any norm $\|\cdot\|$ on $\mathbb{C}^{2}$. As $\varphi^{n}\left(a_{0}\right) \neq h$ for all $n \geq 0$, we see that $\tilde{f}_{0}^{n}(\tilde{a}(0)) \neq$ $(0,0)$ for all $n \geq 0$. Therefore, as described in $\$ 2.1$, we have $\eta=0$ and the function $g_{f, a}$ is given by the formula

$$
g_{f, a}(t)=\lim _{n \rightarrow \infty} \frac{1}{d^{n}} \log \left\|\tilde{f}_{t}^{n}(\tilde{a}(t))\right\|
$$

for $t \in \mathbb{D}^{*}$ [De4, Proposition 3.1].

Set

$$
\Phi:=(P, Q) \text { and } H(z, w):=z-h w
$$

so that $\tilde{f}_{0}=(H P, H Q)$. For all $n \geq 0$, as $\operatorname{deg} \Phi=e>0$, the iteration formula of [De3, Lemma 2.2] states that

$$
\tilde{f}_{0}^{n}=\left(P_{n} \cdot \prod_{k=0}^{n-1}\left(\left(\Phi^{k}\right)^{*} H\right)^{d^{n-k-1}}, Q_{n} \cdot \prod_{k=0}^{n-1}\left(\left(\Phi^{k}\right)^{*} H\right)^{d^{n-k-1}}\right),
$$


where $\Phi^{n}=\left(P_{n}, Q_{n}\right)$, so that

$$
\frac{\log \left\|\tilde{f}_{0}^{n}\right\|}{d^{n}}=\sum_{k=0}^{n-1} \frac{\log \left|\left(\Phi^{k}\right)^{*} H\right|}{d^{k+1}}+\frac{\log \left\|\Phi^{n}\right\|}{d^{n}} \quad \text { on } \mathbb{C}^{2} \backslash\{(0,0)\},
$$

and consequently,

$$
\log \frac{\left\|\tilde{f}_{0} \circ \tilde{f}_{0}^{n}\right\|}{\left\|\tilde{f}_{0}^{n}\right\|^{d}}=\log \frac{\left|\left(\Phi^{n}\right)^{*} H\right|}{\left\|\Phi^{n}\right\|}+\log \frac{\left\|\Phi \circ \Phi^{n}\right\|}{\left\|\Phi^{n}\right\|^{e}} \quad \text { on } \mathbb{C}^{2} \backslash \tilde{f}_{0}^{-n}(0,0) .
$$

Note that $\log \|\Phi\|$ is bounded on the unit sphere in $\mathbb{C}^{2}$, so the last term on the right-hand side of (2.4) is bounded on $\mathbb{C}^{2} \backslash\{(0,0)\}$ uniformly in $n \geq 0$. The first term on the right-hand side of (2.4) is the log of $\left[\varphi^{n}(\cdot), h\right]$, up to scaling of the metric $[\cdot, \cdot]$; therefore, combined with (2.1), we see that there is a constant $C$ so that

$$
\log \frac{\left\|\tilde{f}_{0}\left(\tilde{f}_{0}^{n_{j}}(\tilde{a}(0))\right)\right\|}{\left\|\tilde{f}_{0}^{n_{j}}(\tilde{a}(0))\right\|^{d}}<C+\log \left(r_{n_{j}}\right)=C-n_{j} d^{n_{j}+1}
$$

for all $j$. For all $j$, by continuity of $\tilde{f}_{t}^{n_{j}}(\tilde{a}(t))$ as a map from $\mathbb{D}$ to $\mathbb{C}^{2} \backslash\{(0,0)\}$, there is a radius $\delta_{j} \in(0,1 / 2)$ such that

$$
\sup _{|t| \leq \delta_{j}} \log \frac{\left\|\tilde{f}_{t}\left(\tilde{f}_{t}^{n_{j}}(\tilde{a}(t))\right)\right\|}{\left\|\tilde{f}_{t}^{n_{j}}(\tilde{a}(t))\right\|^{d}} \leq C-n_{j} d^{n_{j}+1} .
$$

On the other hand, we also have from (2.3) that

$$
\begin{aligned}
g_{f, a}(t) & =\log \|\tilde{a}(t)\|+\sum_{k=0}^{\infty} \frac{1}{d^{k+1}} \log \frac{\left\|\tilde{f}_{t}\left(\tilde{f}_{t}^{k}(\tilde{a}(t))\right)\right\|}{\left\|\tilde{f}_{t}^{k}(\tilde{a}(t))\right\|^{d}} \\
& =\log \|\tilde{a}(t)\|+\frac{1}{d^{n_{j}+1}} \log \frac{\left\|\tilde{f}_{t}\left(\tilde{f}_{t}^{n_{j}}(\tilde{a}(t))\right)\right\|}{\left\|\tilde{f}_{t}^{n_{j}}(\tilde{a}(t))\right\|^{d}}+\sum_{k \neq n_{j}} \frac{1}{d^{k+1}} \log \frac{\left\|\tilde{f}_{t}\left(\tilde{f}_{t}^{k}(\tilde{a}(t))\right)\right\|}{\left\|\tilde{f}_{t}^{k}(\tilde{a}(t))\right\|^{d}}
\end{aligned}
$$

for each $j$.

The following is elementary but useful:

Lemma 2.2. Let $F_{t}=\left(P_{t}, Q_{t}\right)$ be any family of homogeneous polynomial maps of degree $d \geq 2$, with coefficients that are bounded holomorphic functions of $t$ in $\mathbb{D}$. Then there is a constant $C$ so that

$$
\frac{\left\|F_{t}(z, w)\right\|}{\|(z, w)\|^{d}} \leq C
$$

for all $(z, w) \in \mathbb{C}^{2} \backslash\{(0,0)\}$ and all $t \in \mathbb{D}$.

Proof. As $F_{t}$ is homogeneous, it suffices to bound its values on the unit sphere in $\mathbb{C}^{2}$. The result follows because the coefficients are bounded uniformly on $\mathbb{D}$.

As a consequence of Lemma 2.2, we can bound all the terms in the final sum of (2.6) from above, uniformly on the disk $\{|t| \leq 1 / 2\}$, and therefore there is a constant $C^{\prime}$ so that

$$
\sup _{|t| \leq 1 / 2} g_{f, a}(t) \leq C^{\prime}+\frac{1}{d^{n_{j}+1}} \log \frac{\left\|\tilde{f}_{t}\left(\tilde{f}_{t}^{n_{j}}(\tilde{a}(t))\right)\right\|}{\left\|\tilde{f}_{t}^{n_{j}}(\tilde{a}(t))\right\|^{d}}
$$


for every $j$. Combined with (2.5), we conclude that there is another constant $C$ so that

$$
\sup _{|t| \leq \delta_{j}} g_{f, a}(t) \leq C-n_{j}
$$

for every $j$. Letting $j \rightarrow \infty$ shows that

$$
\limsup _{t \rightarrow 0} g_{f, a}(t)=-\infty
$$

2.4. Examples with degree $d=2$. Fix any $\theta \in \mathbb{R} \backslash \mathbb{Q}$, and let

$$
\varphi(z)=e^{2 \pi i \theta} z .
$$

Set $a_{0}=1$; the $\omega$-limit set $\omega_{\varphi}\left(a_{0}\right)$ is the unit circle in $\mathbb{C}$. Set $r_{n}=\exp \left(-n 2^{n+1}\right)$ for each $n \in \mathbb{N}$, and define $B_{\varphi}\left(1,\left\{r_{n}\right\}\right)$ as above. Taking any $h \in B_{\varphi}\left(1,\left\{r_{n}\right\}\right)$ and setting $\varepsilon=1$, we define the family $f_{t}$ as in (2.2). Then the potential function $g_{f, a}$ fails to be bounded around $t=0$ for any holomorphic map $a: \mathbb{D} \rightarrow \mathbb{P}^{1}$ with $a(0)=1$.

Note that only the Möbius transformations $\varphi$ which are Möbius (i.e., $\operatorname{PSL}(2, \mathbb{C})-$ ) conjugate to an irrational rotation have recurrent orbits, as needed for the construction described above.

2.5. Examples in degree $>2$, with a marked critical point. Fix an integer $d>2$. For every $\theta \in \mathbb{R}$, the polynomial

$$
\varphi(z)=e^{2 \pi i \theta}\left(z-\frac{e^{2 \pi i \theta}-(d-1)}{(d-1)^{(d-1) /(d-2)}}\right)^{d-1}
$$

of degree $d-1$ has a fixed point with multiplier $e^{2 \pi i \theta}$ and its unique finite critical value at $z=0$. Now fix $\theta$ to be irrational; the critical point

$$
a_{0}:=\frac{e^{2 \pi i \theta}-(d-1)}{(d-1)^{(d-1) /(d-2)}}
$$

of $\varphi$ satisfies $\#\left\{\varphi^{n}\left(a_{0}\right): n \in \mathbb{N}\right\}=\infty$ and $a_{0} \in \omega_{\varphi}\left(a_{0}\right)$ [Ma]. Let

$$
r_{n}=\exp \left(-n d^{n+1}\right)
$$

for each $n \in \mathbb{N}$ and fix any point $h \in B_{\varphi}\left(a_{0},\left\{r_{n}\right\}\right)$. Choose any $\varepsilon \in\left(0,\left|a_{0}-h\right|\right]$ and set

$$
f_{t}(z)=\varphi(z) \cdot \frac{z-h-\varepsilon t}{z-h+\varepsilon t}
$$

which is a rational map on $\mathbb{P}^{1}$ of degree $d$ for all $t \in \mathbb{D}^{*}$. We let

$$
a(t)=a_{0}
$$

for all $t \in \mathbb{D}$, which satisfies $f_{t}^{\prime}(a(t))=0$ for all $t \in \mathbb{D}^{*}$. (This is the reason for requiring the unique finite critical value $\varphi\left(a_{0}\right)$ of $\varphi$ to be 0 .) It follows that $g_{f, a}$ fails to be bounded around $t=0$. 
2.6. Example in degree 2, with a marked critical point. We can produce examples of $(f, a)$ also for quadratic rational maps $f_{t}$ where $a(t)$ parameterizes a critical point of $f_{t}$, though we do not have as much flexibility as in higher degrees. For example, we have:

Lemma 2.3. Suppose $f_{t}(z)=\varphi(z)\left(z-h-t^{n}\right) /\left(z-h+t^{n}\right)$ is a family of quadratic rational maps, for some $h \in \mathbb{C}, n \in \mathbb{N}$, and a rational map $\varphi$ on $\mathbb{P}^{1}$ of degree 1 , and suppose that $c_{1}, c_{2}: \mathbb{D} \rightarrow \mathbb{P}^{1}$ are holomorphic maps parameterizing the two critical points of $f_{t}$. Then

$$
\lim _{t \rightarrow 0} c_{1}(t)=\lim _{t \rightarrow 0} c_{2}(t)=h .
$$

Proof. As $\varphi$ has degree 1, it has no critical points of its own. On the other hand, $\lim _{t \rightarrow 0} f_{t}=\varphi$ locally uniformly on $\mathbb{P}^{1} \backslash\{h\}$, so it must be that $c_{1}(t), c_{2}(t) \rightarrow h$ as $t \rightarrow 0$.

In particular, if we wish to let $a(t)$ parameterize a critical point of $f_{t}$, then necessarily we will have $a(0)=h$, which was not allowed by the construction above.

However, let us fix our decreasing sequence as

$$
r_{n}=\exp \left(-(n-1) 2^{n}\right),
$$

for each $n \in \mathbb{N}$, and now apply the Baire Category Theorem to the space of rotations $z \mapsto e^{2 i \pi \theta} z$ to find

$$
\theta_{0} \in\left(\bigcap_{N \in \mathbb{N}} \bigcup_{n \geq N}\left\{\theta \in \mathbb{R}:\left[1, e^{2 \pi i n \theta}\right]<r_{n}\right\}\right) \backslash \mathbb{Q} .
$$

Then $e^{2 \pi i n \theta_{0}} \neq 1$ for all $n \in \mathbb{N}$, and there is a sequence $n_{j} \rightarrow \infty$ as $j \rightarrow \infty$ such that

for all $j$.

$$
0<\left[1, e^{2 \pi i n_{j} \theta_{0}}\right]<r_{n_{j}}
$$

Now set $\varphi_{0}(z)=e^{2 \pi i \theta_{0}} z$, and

$$
f_{t}(z)=\varphi_{0}(z) \cdot \frac{z-1-t^{2}}{z-1+t^{2}}
$$

for $t \in \mathbb{D}^{*}$. Note that we have used $t^{2}$ here rather than $t$ in (2.2); this is so that we can holomorphically parameterize the critical points of $f_{t}$. Indeed, the critical points of $f_{t}$ are

$$
c_{ \pm}(t)=1-t^{2} \pm \sqrt{2 t^{4}-2 t^{2}}=1-t^{2} \pm i \sqrt{2} t \sqrt{1-t^{2}}
$$

which extend holomorphically on $\mathbb{D}$ by setting $c_{ \pm}(0)=1$. Define the function $a: \mathbb{D} \rightarrow \mathbb{C}$ by either $c_{+}$or $c_{-}$so that $f_{t}$ has the critical value

$$
v(t):=f_{t}(a(t))=e^{2 \pi i \theta_{0}}+O(t) \quad \text { as } t \rightarrow 0,
$$

which also extends holomorphically to $\mathbb{D}$ by setting $v(0):=v_{0}:=e^{2 \pi i \theta_{0}}=\varphi_{0}(1)$. We also set

$$
\tilde{f}_{t}(z, w):=\left(e^{2 \pi i \theta_{0}} z\left(z-\left(1+t^{2}\right) w\right),\left(z-\left(1-t^{2}\right) w\right) w\right)
$$

for all $t \in \mathbb{D}$.

We will work with the pair $(f, v)$. Then, since $\varphi_{0}^{n}\left(v_{0}\right) \neq 1$ for all $n \in \mathbb{N} \cup\{0\}$ and since we also have

$$
0<\left[1, \varphi_{0}^{n_{j}-1}\left(v_{0}\right)\right]<r_{n_{j}}=\exp \left(-\left(n_{j}-1\right) 2^{\left(n_{j}-1\right)+1}\right)
$$


for all $j$, the arguments above go through exactly as before - applied to the sequence $\left\{n_{j}-1\right\}_{j}$ - to show that $g_{f, v}$ fails to be bounded around $t=0$.

Finally, if we set $\tilde{v}(t)=(v(t), 1)$ and $\tilde{a}(t)=(a(t), 1)$, then

$$
\tilde{f}_{t}(\tilde{a}(t))=\left(a(t)-1+t^{2}\right) \tilde{v}(t) .
$$

Note that $a(t)-1+t^{2}= \pm i \sqrt{2} t \sqrt{1-t^{2}}$ on $\mathbb{D}$ so that the function

$$
h(t)=\log \left|a(t)-1+t^{2}\right|-\log |t|
$$

on $\mathbb{D}^{*}$ extends to a harmonic function on the disk $\mathbb{D}$. We have

$$
G_{\tilde{f}}(t, \tilde{v}(t))=g_{f, v}(t)
$$

on $\mathbb{D}^{*}$ from the definitions given in (1.1) and because the pair satisfies the hypotheses for (2.3). Consequently,

$$
\begin{aligned}
G_{\tilde{f}}(t, \tilde{a}(t)) & =\frac{1}{2} G_{\tilde{f}}\left(t, \tilde{f}_{t}(\tilde{a}(t))\right) \\
& =\frac{1}{2}\left(G_{\tilde{f}}(t, \tilde{v}(t))+\log \left|a(t)-1+t^{2}\right|\right) \\
& =\frac{1}{2} g_{f, v}(t)+\frac{1}{2} h(t)+\frac{1}{2} \log |t|
\end{aligned}
$$

so that, by the definition of $g_{f, a}$ in (1.1), we have $\eta=1 / 2$ and

$$
g_{f, a}(t)=\frac{1}{2} g_{f, v}(t)+\frac{1}{2} h(t)
$$

on $\mathbb{D}^{*}$. We conclude that the function $g_{f, a}$ also fails to be bounded near $t=0$.

\section{LYAPUNOV EXPONENTS AND THE BIFURCATION CURRENT}

The Lyapunov exponent of an individual rational map $f$ on $\mathbb{P}^{1}$ of degree $>1$, with respect to its unique measure $\mu_{f}$ of maximal entropy on $\mathbb{P}^{1}$, is the positive and finite quantity

$$
L(f)=\int_{\mathbb{P}^{1}} \log \left|f^{\prime}\right| d \mu_{f}
$$

where $|\cdot|$ is any choice of metric on the tangent bundle of $\mathbb{P}^{1}$.

Let $f_{t}$ be a holomorphic family of rational maps on $\mathbb{P}^{1}$ of degree $d>1$ parameterized by $\mathbb{D}^{*}$ whose coefficients extend to meromorphic functions on $\mathbb{D}$. If all the critical points of $f_{t}$ are parameterized by holomorphic maps $c_{1}, \ldots, c_{2 d-2}: \mathbb{D} \rightarrow \mathbb{P}^{1}$, then

$$
L\left(f_{t}\right)=h(t)+\sum_{j=1}^{2 d-2} g_{f, c_{j}}(t)
$$

on $\mathbb{D}^{*}$, for a harmonic function $h$ on $\mathbb{D}^{*}$ satisfying $h(t)=O(\log |t|)$ as $t \rightarrow 0$ [De2, Theorem 1.4], [Fa2, Theorem C]. By the symmetry in the critical points in (3.1), this formula holds even if the critical points cannot be holomorphically parameterized on $\mathbb{D}^{*}$. The bifurcation current associated to the family $f_{t}$ can be given by

$$
T_{\mathrm{bif}}:=\frac{1}{2 \pi} \Delta L\left(f_{t}\right)
$$

on $\mathbb{D}^{*}$, in the sense of distributions; the original definition of $T_{\mathrm{bif}}$ in [De1] was based on the right-hand side of (3.1). From [De2, Theorem 1.1], the support of $T_{\text {bif }}$ is equal to the bifurcation locus of the family $f_{t}$ in the sense of [MSS, Ly]. 
In particular, because the sum $\sum_{j} g_{f, c_{j}}$ in (3.1) is $o(\log |t|)$ near $t=0$, we see that the bifurcation current $T_{\text {bif }}$ has a bounded potential if and only if the sum $\sum_{j} g_{f, c_{j}}$ is bounded near $t=0$.

Proof of Theorem 1.1. We give examples in an arbitrary degree $>1$. First, let $f_{t}$ be the holomorphic family of quadratic rational maps on $\mathbb{P}^{1}$ parameterized by $\mathbb{D}^{*}$ described in $\$ 2.6$. As we have seen, neither of the functions $g_{f, c_{ \pm}}$extend continuously to $\mathbb{D}$; indeed, both tend to $-\infty$ as $t \rightarrow 0$. Hence by (3.1), the bifurcation current for the family $f_{t}$ fails to have a potential bounded around $t=0$.

Next, let $f_{t}$ be the holomorphic family of rational maps on $\mathbb{P}^{1}$ of degree $d>2$ parameterized by $\mathbb{D}^{*}$, described in $\$ 2.5$. As we have seen, the constant map $a(t) \equiv$ $a_{0}$ on $\mathbb{D}$ satisfies $f_{t}^{\prime}(a(t))=0$ for every $t \in \mathbb{D}^{*}$, and the function $g_{f, a}$ tends to $-\infty$ as $t \rightarrow 0$. Taking an at most finitely ramified holomorphic covering $\pi: \mathbb{D}^{*} \rightarrow \mathbb{D}^{*}$ if necessary, all the critical points of $f_{\pi(s)}$ are parameterized by holomorphic maps $c_{1}, \ldots, c_{2 d-2}: \mathbb{D} \rightarrow \mathbb{P}^{1}$. We may assume the points are labeled so that $c_{1}=a \circ \pi$ on $\mathbb{D}$. By the formula (2.3) for $g_{f, c_{1}}$, we have $g_{f_{\pi(\cdot)}, c_{1}}(s)=g_{f, a}(\pi(s))$ on $\mathbb{D}^{*}$. On the other hand, for every $j \in\{2, \ldots, 2 d-2\}$, the function $g_{f_{\pi(\cdot)}, c_{j}}$ is bounded from above on $\{0<|s| \leq r\}$ for every $r \in(0,1)$, by Lemma 2.1. Hence the sum $\sum_{j} g_{f_{\pi(\cdot)}, c_{j}}(s)$ tends to $-\infty$ as $s \rightarrow 0$. Therefore, the bifurcation current associated to the family $f_{t}$ fails to have a potential bounded around $t=0$.

\section{Limitations of the CONSTRUCtion}

To find the examples of Section 2, we used a rational map $\varphi \in \mathbb{C}(z)$ of degree $\geq 1$ and points $a_{0}, h \in \mathbb{P}^{1}(\mathbb{C})$ such that

$$
0<\left[\varphi^{n_{j}}\left(a_{0}\right), h\right]<r_{n_{j}}
$$

in the chordal metric $[\cdot, \cdot]$, along a sequence $n_{j} \rightarrow \infty$, with $\left(r_{n}\right)$ chosen so that

$$
\lim _{n \rightarrow \infty} \frac{\log r_{n}}{d^{n}}=-\infty
$$

Combining (2.7) with (2.5) guaranteed that $\lim _{t \rightarrow 0} g_{f, a}(t)=-\infty$. Looking carefully at the estimates, we see that the orbit $\left\{\varphi^{n}\left(a_{0}\right)\right\}$ needs only to satisfy a weaker divergence condition

$$
\sum_{n=0}^{\infty} \frac{\log \left[\varphi^{n}\left(a_{0}\right), h\right]}{d^{n}}=-\infty,
$$

with $\varphi^{n}\left(a_{0}\right) \neq h$ for all $n \in \mathbb{N} \cup\{0\}$, to achieve our conclusion with this method.

As observed in the Introduction, the function $g_{f, a}$ will always extend continuously to $\mathbb{D}$ when $f_{t}$ is a family of polynomials, by [FG2, Main Theorem]. Here we explain explicitly why our construction breaks down for polynomials.

Proposition 4.1. The construction of Section 2 cannot produce any pair $(f, a)$ such that for every $t \in \mathbb{D}^{*}, f_{t}$ is Möbius conjugate to a polynomial.

Proof. Suppose that $f_{t}$ is a holomorphic family of rational maps of degree $d>1$ parameterized by $\mathbb{D}^{*}$ such that for every $t \in \mathbb{D}^{*}$, there exists $A_{t} \in \operatorname{PSL}(2, \mathbb{C})$ such that $A_{t} \circ f_{t} \circ A_{t}^{-1}$ is a polynomial, and that $\lim _{t \rightarrow 0} f_{t}=\varphi$ locally uniformly on $\mathbb{P}^{1} \backslash\{h\}$ for some $h \in \mathbb{P}^{1}$ and some $\varphi \in \mathbb{C}(z)$ of degree $d-1(>0)$. For every $t \in \mathbb{D}^{*}$, the point $p_{t}:=A_{t}^{-1}(\infty)$ is a superattracting fixed point of $f_{t}$ for which $\operatorname{deg}_{p_{t}} f_{t}=d$. We first claim that $\lim _{t \rightarrow 0} p_{t}=h$; otherwise, there is a sequence $\left(t_{j}\right)$ 
in $\mathbb{D}^{*}$ tending to 0 as $j \rightarrow \infty$ such that there is the limit $p:=\lim _{j \rightarrow \infty} p_{t_{j}} \in \mathbb{P}^{1} \backslash\{h\}$. By the locally uniform convergence $\lim _{t \rightarrow 0} f_{t}=\varphi$ on $\mathbb{P}^{1} \backslash\{h\}$, $\operatorname{deg} \varphi>0$, and the Argument Principle, this $p$ must be a superattracting fixed point of $\varphi$ for which $\operatorname{deg}_{p} \varphi=d$, contradicting $\operatorname{deg} \varphi=d-1$. We next claim that $\varphi^{-1}(h)=\{h\}$; for, if there is a point $q \in \mathbb{P}^{1} \backslash\{h\}$ for which $\varphi(q)=h$, then by the first claim, the locally uniform convergence $\lim _{t \rightarrow 0} f_{t}=\varphi$ on $\mathbb{P}^{1} \backslash\{h\}, \operatorname{deg} \varphi>0$, and the Argument Principle, for any $t \in \mathbb{D}^{*}$ close enough to 0 , there must exist a point $q_{t} \in \mathbb{P}^{1} \backslash\left\{p_{t}\right\}$ (near $q$ ) for which $f_{t}\left(q_{t}\right)=p_{t}$, contradicting $\operatorname{deg} f_{t}=d$.

Suppose $a: \mathbb{D} \rightarrow \mathbb{P}^{1}$ is any holomorphic map with $a(0)=: a_{0} \neq h$. If $d>2$ so that $\operatorname{deg} \varphi=d-1>1$, then by the second claim, we have a constant $C<0$ so that

$$
\log \left[\varphi^{n}\left(a_{0}\right), h\right] \geq C \cdot(d-1)^{n}
$$

for all $n \in \mathbb{N}$. If $d=2$ so that $\operatorname{deg} \varphi=d-1=1$, then by the second claim above, the orbit $\left\{\varphi^{n}\left(a_{0}\right)\right\}$ can accumulate to $h$ and satisfy $\varphi^{n}\left(a_{0}\right) \neq h$ for any $n \geq 0$ only if $h$ is an attracting or parabolic fixed point of $\varphi$. Hence we still have a constant $C<0$ so that

$$
\log \left[\varphi^{n}\left(a_{0}\right), h\right] \geq C \cdot n
$$

for all $n \in \mathbb{N}$. Therefore in both cases, the points $a_{0}, h \in \mathbb{P}^{1}$ cannot satisfy (4.1).

Working over the field $\mathbb{C}$ of complex numbers allowed us to exploit the Baire Category Theorem in our construction. In fact, the construction is impossible over a field such as $\overline{\mathbb{Q}}$.

Proposition 4.2. The construction of Section 2 cannot produce any pair $(f, a)$ such that the map $\varphi$ and points $a_{0}$ and $h$ are simultaneously defined over $\overline{\mathbb{Q}}$.

Proof. Suppose $f_{t}$ is any holomorphic family of rational maps of degree $d>1$ parameterized by $\mathbb{D}^{*}$ such that $\lim _{t \rightarrow 0} f_{t}=\varphi$ locally uniformly on $\mathbb{P}^{1} \backslash\{h\}$, for some $\varphi \in \overline{\mathbb{Q}}(z)$ of degree $d-1$ and some $h \in \mathbb{P}^{1}(\overline{\mathbb{Q}})$. Fix any point $a_{0} \in \mathbb{P}^{1}(\overline{\mathbb{Q}})$ such that $\varphi^{n}\left(a_{0}\right) \neq h$ for all $n \geq 0$.

Suppose first that $d>2$ so that $\operatorname{deg} \varphi=d-1>1$. If there is $A \in \operatorname{PSL}(2, \overline{\mathbb{Q}})$ such that either $A \circ \varphi \circ A^{-1}$ or $A \circ \varphi^{2} \circ A^{-1}$ is a polynomial and that $A(h)=\infty$, then we have a constant $C<0$ so that

$$
\log \left[\varphi^{n}\left(a_{0}\right), h\right] \geq C(d-1)^{n}
$$

for all $n \in \mathbb{N}$. Otherwise, by [Si1, Theorem E], which uses the Roth theorem, we have the stronger result that

$$
\log \left[\varphi^{n}\left(a_{0}\right), h\right]=o\left((d-1)^{n}\right)
$$

as $n \rightarrow \infty$. Therefore, in both cases, $\varphi, a_{0}$, and $h$ cannot satisfy (4.1).

Now suppose that $d=2$ so that $\operatorname{deg} \varphi=d-1=1$. Note that the orbit $\left\{\varphi^{n}\left(a_{0}\right)\right\}$ can accumulate to $h$ and satisfy $\varphi^{n}\left(a_{0}\right) \neq h$ for any $n \geq 0$ only if either $h$ is an attracting or parabolic fixed point of $\varphi\left(\right.$ in $\left.\mathbb{P}^{1}(\overline{\mathbb{Q}})\right)$ or there exists $A \in \operatorname{PSL}(2, \overline{\mathbb{Q}})$ such that $A \circ \varphi \circ A^{-1}$ is an irrational rotation $z \mapsto \lambda z$, where $\lambda$ is not a root of unity, $\lambda \in \overline{\mathbb{Q}}$, and $|\lambda|=1$, with $|A(h)|=\left|A\left(a_{0}\right)\right|=1$. In the former case, we have a constant $C<0$ so that $\log \left[\varphi^{n}\left(a_{0}\right), h\right] \geq C \cdot n$ for all $n \in \mathbb{N}$. So $\varphi, a_{0}$, and $h$ cannot satisfy (4.1).

In the latter case, we claim that we still have a constant $C<0$ such that

$$
\log \left[\varphi^{n}\left(a_{0}\right), h\right] \geq C \cdot n
$$


for all $n \in \mathbb{N}$; since $A \in \operatorname{PSL}(2, \overline{\mathbb{Q}})$ is biLipschitz with respect to $[\cdot, \cdot]$, we can assume that $\varphi$ is an irrational rotation $z \mapsto \lambda z$, where $\lambda$ is not a root of unity, $\lambda \in \overline{\mathbb{Q}}$, and $|\lambda|=1$, with $h, a_{0} \in \overline{\mathbb{Q}}$ and $|h|=\left|a_{0}\right|=1$. Fix a number field $K$ so that $\lambda, a_{0}, h \in K$, and denote by $M_{K}$ the set of all places (i.e., equivalence classes of nontrivial either archimedean or nonarchimedean absolute values) of $K$. Recall that there are a family $\left(N_{v}\right)_{v \in M_{K}}$ in $\mathbb{N}$ and a family $\left(|\cdot|_{v}\right)_{v \in M_{K}}$ of representatives $|\cdot|_{v}$ of places $v$ such that for every $x \in K^{*},|x|_{v}=1$ for all but finitely many $v \in M_{K}$ and $\prod_{v \in M_{K}}|x|_{v}^{N_{v}}=1$. Then by the (strong) triangle inequality, we can choose a family of real numbers $C_{v} \geq 1, v \in M_{K}$, such that $\left|\lambda^{n}-h / a_{0}\right|_{v} \leq C_{v}^{n}$ for any $v \in M_{K}$ and any $n \in \mathbb{N}$ and that $C_{v}=1$ for all but finitely many $v \in M_{K}$. We also note that $\lambda^{n} a_{0}=\varphi^{n}\left(a_{0}\right) \neq h$ for all $n \in \mathbb{N}$. Hence for every $v_{0} \in M_{K}$ and every $n \in \mathbb{N}$, we have $\left|\lambda^{n}-h / a_{0}\right|_{v_{0}} \geq\left(\prod_{v \in M_{K}} C_{v}^{-N_{v}}\right)^{n}$. In particular, recalling that $[z, w]=|z-w|[z, \infty][w, \infty]$ on $\mathbb{C} \times \mathbb{C}$, there is a constant $C<0$ such that

$$
\log \left[\varphi^{n}\left(a_{0}\right), h\right]=\log \left|\lambda^{n}-h / a_{0}\right|-\log 2 \geq C \cdot n
$$

for all $n \in \mathbb{N}$. So the claim holds, and $\varphi, a_{0}$, and $h$ cannot satisfy (4.1).

\section{ACKNOWLEDGMENTS}

We would like to thank Charles Favre, Thomas Gauthier, and the anonymous referee for helpful comments and suggestions.

\section{REFERENCES}

[BD] Matthew Baker and Laura De Marco, Special curves and postcritically finite polynomials, Forum Math. Pi 1 (2013), e3, 35. MR3141413

[BG] François Berteloot and Thomas Gauthier, On the geometry of bifurcation currents for quadratic rational maps, Ergodic Theory Dynam. Systems 35 (2015), no. 5, 1369-1379. MR.3365726

[Bu] Xavier Buff, Courants dynamiques pluripolaires (French, with English and French summaries), Ann. Fac. Sci. Toulouse Math. (6) 20 (2011), no. 1, 203-214. MR2830397

[De1] Laura DeMarco, Dynamics of rational maps: a current on the bifurcation locus, Math. Res. Lett. 8 (2001), no. 1-2, 57-66. MR.1825260

[De2] Laura DeMarco, Dynamics of rational maps: Lyapunov exponents, bifurcations, and capacity, Math. Ann. 326 (2003), no. 1, 43-73. MR1981611

[De3] Laura DeMarco, Iteration at the boundary of the space of rational maps, Duke Math. J. 130 (2005), no. 1, 169-197. MR2176550

[De4] Laura DeMarco, Bifurcations, intersections, and heights, Algebra Number Theory 10 (2016), no. 5, 1031-1056. MR3531361

[DM] L. DeMarco and N.M. Mavraki, Variation of canonical height and equidistribution. Preprint, arXiv:1701.07947 [math.NT].

[DG] Jeffrey Diller and Vincent Guedj, Regularity of dynamical Green's functions, Trans. Amer. Math. Soc. 361 (2009), no. 9, 4783-4805. MR2506427

[DF] Romain Dujardin and Charles Favre, Distribution of rational maps with a preperiodic critical point, Amer. J. Math. 130 (2008), no. 4, 979-1032. MR2427006

[Fa1] Charles Favre, Points périodiques d'applications birationnelles de $\mathbf{P}^{2}$ (French, with English and French summaries), Ann. Inst. Fourier (Grenoble) 48 (1998), no. 4, 999-1023. MR 1656005

[Fa2] Charles Favre, Degeneration of endomorphisms of the complex projective space in the hybrid space. Preprint, arXiv:1611.08490v1 [math.DS].

[FG1] Charles Favre and Thomas Gauthier, Classification of special curves in the space of cubic polynomials. To appear, Int. Math. Res. Not., doi 10.1093/imrn/rnw245.

[FG2] Charles Favre and Thomas Gauthier, Continuity of the Green function in meromorphic families of polynomials. Preprint, arXiv:1706.04676v2 [math.DS]. 
[FS] John Erik Fornæss and Nessim Sibony, Complex dynamics in higher dimensions, Complex potential theory, Notes partially written by Estela A. Gavosto, (Montreal, PQ, 1993), NATO Adv. Sci. Inst. Ser. C Math. Phys. Sci., vol. 439, Kluwer Acad. Publ., Dordrecht, 1994, pp. 131-186. MR.1332961

[GY] Dragos Ghioca and Hexi Ye, A Dynamical Variant of the André-Oort Conjecture. To appear, Int. Math. Res. Not., doi 10.1093/imrn/rnw314.

[HP] John H. Hubbard and Peter Papadopol, Superattractive fixed points in $\mathbf{C}^{n}$, Indiana Univ. Math. J. 43 (1994), no. 1, 321-365. MR1275463

[JR] Mattias Jonsson and Paul Reschke, On the complex dynamics of birational surface maps defined over number fields. Preprint, arXiv:1505.03559 [math.DS].

[Ly] M. Yu. Lyubich, Some typical properties of the dynamics of rational mappings (Russian), Uspekhi Mat. Nauk 38 (1983), no. 5(233), 197-198. MR.718838

[MSS] R. Mañé, P. Sad, and D. Sullivan, On the dynamics of rational maps, Ann. Sci. École Norm. Sup. (4) 16 (1983), no. 2, 193-217. MR732343

[Ma] Ricardo Mañé, On a theorem of Fatou, Bol. Soc. Brasil. Mat. (N.S.) 24 (1993), no. 1, 1-11. MR 1224298

[Si1] Joseph H. Silverman, Integer points, Diophantine approximation, and iteration of rational maps, Duke Math. J. 71 (1993), no. 3, 793-829. MR1240603

[Si2] Joseph H. Silverman, Variation of the canonical height on elliptic surfaces. II. Local analyticity properties, J. Number Theory 48 (1994), no. 3, 291-329. MR1293864

Department of Mathematics, Northwestern University, 2033 Sheridan Road, Evanston, ILLINOIS 60208

Email address: demarco@math.northwestern.edu

Division of Mathematics, Kyoto Institute of Technology, Sakyo-ku, Kyoto 606-8585, JAPAN

Email address: okuyama@kit.ac.jp 